\title{
Detection and Grading of Endolymphatic Hydrops in Menière Disease Using MR Imaging
}

\author{
K. Baráth, B. Schuknecht, A. Monge Naldi, T. Schrepfer, C.J. Bockisch, and S.C.A. Hegemann
}

\begin{abstract}
BACKGROUND AND PURPOSE: Endolymphatic hydrops has been recognized as the underlying pathophysiology of Menière disease. We used 3T MR imaging to detect and grade endolymphatic hydrops in patients with Menière disease and to correlate MR imaging findings with the clinical severity.
\end{abstract}

\begin{abstract}
MATERIALS AND METHODS: MR images of the inner ear acquired by a 3D inversion recovery sequence 4 hours after intravenous contrast administration were retrospectively analyzed by 2 neuroradiologists blinded to the clinical presentation. Endolymphatic hydrops was classified as none, grade I, or grade II. Interobserver agreement was analyzed, and the presence of endolymphatic hydrops was correlated with the clinical diagnosis and the clinical Menière disease score.

RESULTS: Of 53 patients, we identified endolymphatic hydrops in $90 \%$ on the clinically affected and in $22 \%$ on the clinically silent side. Interobserver agreement on detection and grading of endolymphatic hydrops was 0.97 for cochlear and 0.94 for vestibular hydrops. The average MR imaging grade of endolymphatic hydrops was $1.27 \pm 0.66$ for 55 clinically affected and $0.65 \pm 0.58$ for 10 clinically normal ears. The correlation between the presence of endolymphatic hydrops and Menière disease was 0.67 . Endolymphatic hydrops was detected in $73 \%$ of ears with the clinical diagnosis of possible, $100 \%$ of probable, and $95 \%$ of definite Menière disease.
\end{abstract}

CONCLUSIONS: MR imaging supports endolymphatic hydrops as a pathophysiologic hallmark of Menière disease. High interobserver agreement on the detection and grading of endolymphatic hydrops and the correlation of MR imaging findings with the clinical score recommend MR imaging as a reliable in vivo technique in patients with Menière disease. The significance of MR imaging detection of endolymphatic hydrops in an additional $22 \%$ of asymptomatic ears requires further study.

ABBREVIATIONS: $\mathrm{EH}=$ endolymphatic hydrops, $\mathrm{MD}=$ Menière disease; $3 \mathrm{D}-\mathrm{IR}=3 \mathrm{D}$ real inversion recovery

A ccording to the 1985 American Academy of OtolaryngologyHead and Neck Surgery Committee on Hearing and Equilibrium guidelines, Menière disease (MD) is defined by $\geq 2$ definitive spontaneous episodes of vertigo 20 minutes or longer, audiometrically documented hearing loss on at least 1 occasion, and tinnitus or aural fullness. ${ }^{1}$ In 1995, a clinical diagnostic scale was added with the categories possible, probable, definite, and certain, ${ }^{2}$ with "certain" defined as definite disease plus histo-

Received August 30, 2013; accepted after revision November 20.

From the Medizinisches Radiologisches Institut (MRI Bethanien/Bahnhofplatz/ Stadelhofen) Zurich (K.B., B.S.), Zurich, Switzerland; Department of Oto-RhinoLaryngology (A.M.N., T.S.), University Hospital of Zurich, Zurich, Switzerland; and Departments of Neurology, Ophthalmology and Oto-Rhino-Laryngology (C.J.B.) and Oto-Rhino-Laryngology, Interdisciplinary Center for Vertigo and Balance Disorders (S.C.A.H.), University Hospital of Zurich, Zurich, Switzerland.

B. Schuknecht shared first authorship with K. Baráth.

Please address correspondence to Krisztina Baráth, Medizinisches Radiologisches Institut (MRI Bethanien/Bahnhofplatz/Stadelhofen) Zurich, Toblerstr 51, 8044 Zurich, Switzerland; e-mail: barath.krisztina@hin.ch

http://dx.doi.org/10.3174/ajnr.A3856 pathologic confirmation. It is universally agreed that the pathogenesis of MD consists of endolymphatic hydrops (EH), but a simple cause-effect relation between $\mathrm{EH}$ and clinical symptoms is not present. Moreover, EH appears to be an end point of different etiologies such as trauma, ${ }^{2}$ viral infection and autoimmune processes, ${ }^{3}$ electrolyte imbalance, ${ }^{4}$ and cellular channelopathies. ${ }^{5}$ Histopathology has provided evidence that not every individual with $\mathrm{EH}$ presents with symptoms of $\mathrm{MD}^{6-8}$ and not every individual with the clinical diagnosis of MD has EH. ${ }^{9-12}$ Only recently has MR imaging enabled depiction of $\mathrm{EH},{ }^{13}$ opening a window for in vivo confirmation of $\mathrm{EH}$. The purpose of our study was to assess the degree of $\mathrm{EH}$ in 53 patients with MD and to correlate the MR imaging findings obtained by a specific protocol with the certitude of clinical diagnosis.

\section{MATERIALS AND METHODS}

From June 2012 until April 2013, sixty-three patients, 27-72 years of age, female/male $=21: 42$, with the clinical diagnosis of definite, 

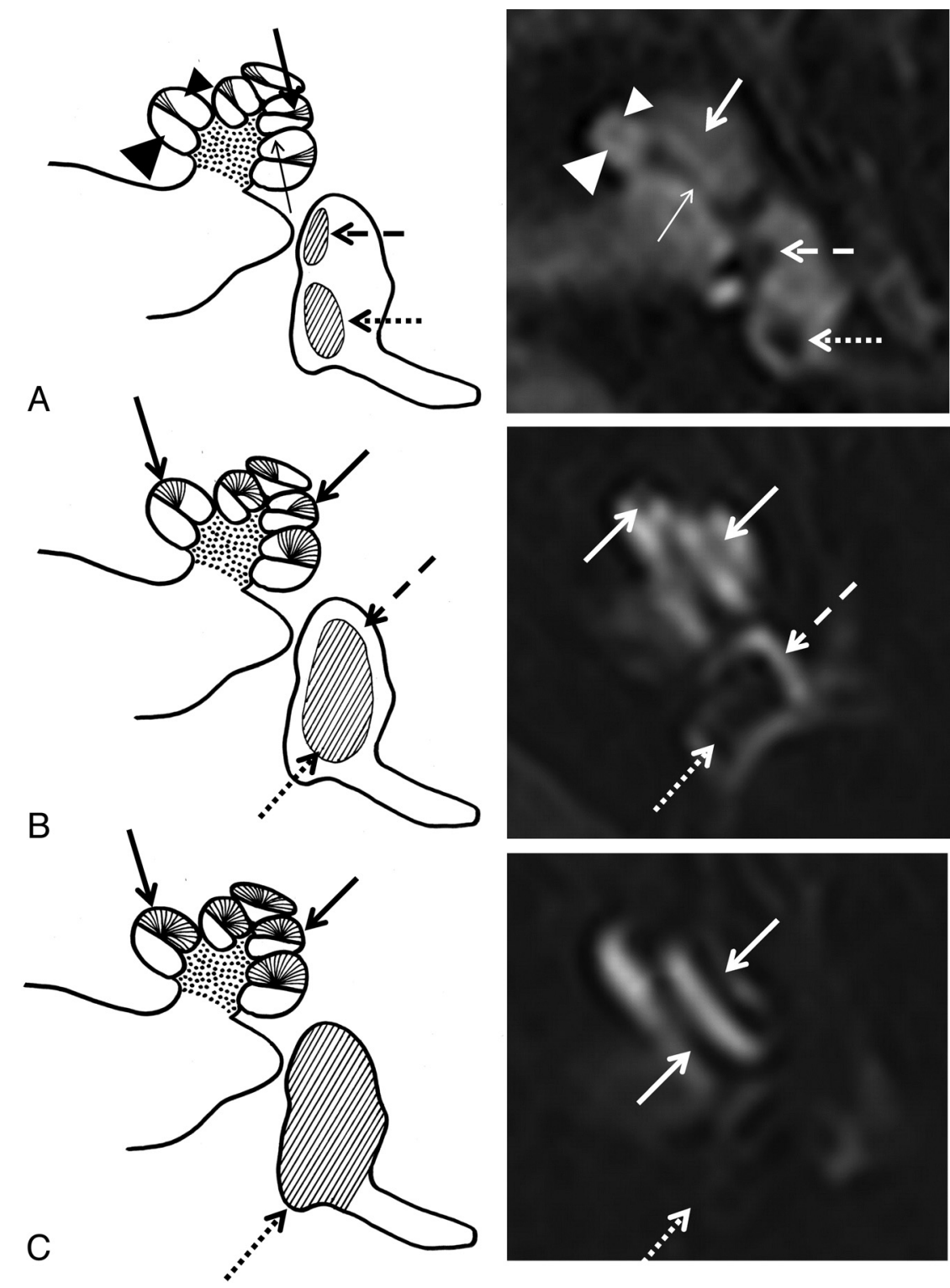

FIG 1. A, Normal labyrinth: interscalar septum (thin arrow), scala tympani (large arrowhead), osseous spiral lamina/cochlear duct (thick arrow), scala vestibuli (small arrowhead), saccule (dashed arrow), and utricle (dotted arrow). B, Cochlear hydrops grade I with irregular dilation and partial obstruction of the scala vestibuli (arrows). In vestibular hydrops grade I, dilation of the endolymphatic space (dotted arrow) encompasses $>50 \%$ of the vestibulum. A circular perilymphatic space (dashed arrow) remains visible. C, Cochlear hydrops grade II with total obliteration of the scala vestibuli (arrows). In vestibular hydrops grade II, dilation of the endolymphatic space leads to effacement of the perilymphatic space (dotted arrow).

possible, or probable MD were referred for 3T MR imaging of the temporal bone to demonstrate $\mathrm{EH}$ and to exclude other causes of vertigo and hearing loss such as vestibular schwannoma. Nine patients with motion artifacts and 1 patient with the MR imaging diagnosis of hemorrhagic labyrinthitis were excluded from analysis. With institutional approval for the study and patient informed consent, the MR imaging data of the remaining 53 patients (106 ears) were retrospectively analyzed.

All patients underwent 3T MR imaging of the temporal bone by using a 32-channel phased array coil to rule out schwannoma or other causes of the symptoms. Following a delay of 4 hours after intravenous contrast administration (Gadovist; BayerSchering Pharma, Berlin, Germany; $1.0 \mathrm{mmol} / \mathrm{mL}$ at a dose of 0.2 $\mathrm{mmol} / \mathrm{kg}$ ), a 3D real inversion recovery (3D-IR) sequence ${ }^{13}$ was performed with the following parameters: FOV, 190 $\mathrm{mm}$; section thickness, $0.8 \mathrm{~mm}$; TR, $6000 \mathrm{~ms}$; TE, $177 \mathrm{~ms}$; number of excitations, 1; TI, $2000 \mathrm{~ms}$; flip angle, $180^{\circ}$; matrix, $384 \times 384$; bandwidth, $213 \mathrm{~Hz} /$ pixel; turbo factor, 27; scan time, 15 minutes.

The MR images were qualitatively analyzed by 2 experienced neuroradiologists (K.B. and B.S.) blinded to the side, uni- or bilaterality of symptoms and the clinical score of MD.

On the basis of previous histopathologic observations, ${ }^{14} \mathrm{EH}$ was categorized as none (Fig $1 A$ ), grade I (Fig $1 B$ ), and grade II (Fig 1C). Hydrops of the cochlea and vestibule was separately assessed by visual comparison of the relative areas of the nonenhanced endolymphatic space versus the contrastenhanced perilymph space.

Statistical analysis for interobserver agreement on detecting and grading $\mathrm{EH}$ was performed by using the Cohen $\kappa$ test. To test for independence of MR imaging and clinical results, we used the Pearson $\chi^{2}$ test (when the clinical grading was simplified to normal and abnormal ears), and the Fisher exact test, when the clinical score was 4 levels (normal ears and ears with possible, probable, and definite MD). All tests were performed in R (Version 2.14.2; http:// www.r-project.org/) and RStudio (Version 0.97; http://www.rstudio.com).

\section{RESULTS}

\section{Normal MR Imaging Findings}

On the delayed 3D-IR sequence, the normal cochlea displays the interscalar septum, scala tympani, osseous spiral lamina/cochlear duct, and scala vestibuli (Fig $1 A$ ). In the normal vestibule, the added surface areas of the saccule and utricle are less than half the area of the vestibule at the midmodiolar level (Fig $1 A$ ).

\section{Grading of EH}

Grade I cochlear hydrops was defined as mild dilation of the nonenhancing cochlear duct, sparing parts of the enhancing perilymph of the scala vestibuli (Fig $1 B$ ). Grade I vestibular hydrops presented as distention of the endolymph space of the saccule or utricle or both, with the perilymphatic space still visible along the periphery of the bony vestibule (Fig $1 B$ ).

In grade II cochlear hydrops, the scala vestibuli was uniformly obstructed by the maximally distended cochlear duct 
3D-IR sequence

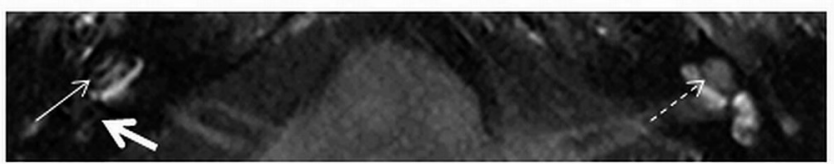

Below midmodiolarlevel
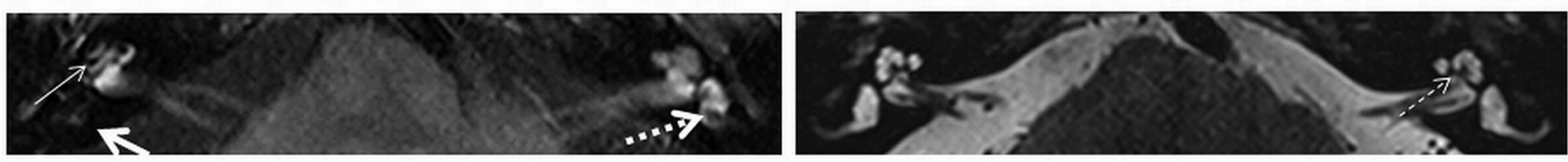

Midmodiolarlevel
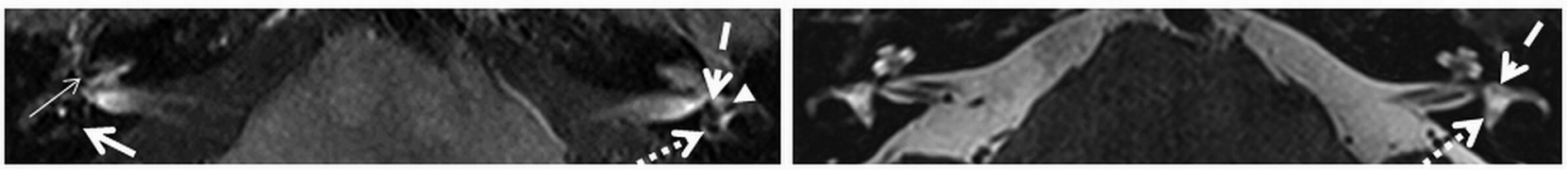

Above midmodiolarlevel

FIG 2. A 3D-IR sequence depicts cochlear EH grade II (thin arrow) and vestibular EH grade II (thick arrow) on the right. EH is not visible on the corresponding 3D T2-weighted spatial and chemical-shift encoded excitation (SPACE) sequence. No EH on the normal left side is seen. Normal anatomy is shown at different levels (below the midmodiolar, midmodiolar, and above the midmodiolar sections) on the 3D-IR (0.8 mm) and 3D heavily T2-weighted SPACE $(0.4 \mathrm{~mm})$ sequence: interscalar septum (thin dashed arrow), anterior ampulla (thick dashed arrow), utricle/common crus (thick dotted arrow), and the lateral ampulla (arrowhead).

(Figs $1 C$ and 2). In grade II vestibular hydrops, the bony vestibule was entirely encompassed by the dilated endolymphatic spaces (Figs $1 C$ and 2).

EH was not visible on the 3D heavily T2-weighted spatial and chemical-shift encoded excitation sequence obtained at a $0.4-\mathrm{mm}$ section thickness (Fig 2).

\section{MR Imaging Findings of $\mathrm{EH}$}

Cochlear hydrops was present in 53 ears (grade I in 35 and grade II in 18 cases), and vestibular hydrops was detected in 56 ears (grade I in 30 and grade II in 26 instances). Cohen $\kappa$ test for interobserver agreement was 0.97 for cochlear findings and 0.94 for the vestibule (normal and abnormal). The average MR imaging grading of $\mathrm{EH}$ was $1.27 \pm 0.66$ for 55 clinically affected ears ( 55 cochleae and 55 vestibules) and $0.65 \pm 0.581$ for 10 clinically normal sides ( 10 cochleae and 10 vestibules).

\section{Correlation of Imaging and Clinical Diagnosis}

Ninety percent (55/61) of clinically diseased ears had EH on MR imaging, whereas $78 \%$ (35/45) of the clinically normal ears had no $\mathrm{EH}$ on MR imaging (Fig $3 A$ ). The results proved significantly different from chance (Pearson $\chi^{2}$ test with the Yates continuity correction, $\left.\chi^{2}=47.5754, d f=1, P<.001\right)$. Conversely, $22 \%$ (10/45) of clinically normal ears showed EH on MR imaging, and $10 \%(6 / 61)$ of ears with a clinical diagnosis of MD did not show $\mathrm{EH}$.

Of the 10 ears with asymptomatic unilateral $\mathrm{EH}, \mathrm{MR}$ imaging in 9 ears $(92 \%)$ depicted grade I EH in either the cochlea $(n=3)$, the vestibule $(n=5)$, or both $(n=1)$. Only in 1 case was grade II vestibular EH present in addition to a grade I cochlear EH.

The correlation between the presence of $\mathrm{EH}$ and the clinical score (normal ears and ears with possible, probable, and definite MD) was 0.67 (Spearman $\rho, P<.001$ ). EH was depicted in $73 \%$ $(11 / 15)$ of ears with the clinical diagnosis of possible MD, in $100 \%$
(3/3) with probable MD, and in 95\% (41/43) of sides with definite MD (Fig 3B). These percentages are significantly different from chance (Fisher Exact Test for Count Data, $P$ value $<.001$ ).

\section{DISCUSSION}

\section{MR Imaging Visualization of EH}

Since the first publication of MR imaging visualization of $\mathrm{EH}$ in an animal study in $2001^{15}$ and MR imaging demonstration of $\mathrm{EH}$ in patients with MD following intratympanic injection in 2007, ${ }^{16}$ numerous studies have tried to visualize $\mathrm{EH}$ by using different routes of administration of contrast media, such as intratympanic $^{16}$ versus intravenous ${ }^{17-20}$ and by altering intravenous dosage regimens (single, ${ }^{13,17}$ double, ${ }^{18}$ and triple ${ }^{21}$ dose). Additional variations included technical parameters such as the number of receive channels of the head coil $\left(8,{ }^{22} 12,{ }^{23}\right.$ and $\left.32,{ }^{17}\right)$ and a variable choice of sequences such as 3D-FLAIR, ${ }^{23,24}$ heavily T2weighted 3D-FLAIR, ${ }^{24}$ and $3 \mathrm{D}$-IR sequences. ${ }^{13}$ On the basis of previous demonstration of the feasibility to separate the endoand perilymphatic space 24 hours after intratympanic gadolinium injection by a 3D-IR sequence ${ }^{13}$ and the observation that perilymphatic enhancement occurs 4 hours after intravenous contrast administration, ${ }^{21,25}$ our protocol comprised a $3 \mathrm{D}$-IR sequence obtained 4 hours following intravenous injection of contrast media.

The intravenous route of contrast administration is also less invasive and renders perilymph enhancement independent of the status of the round window membrane. ${ }^{26,27}$ In a comparative study in patients with $\mathrm{MD}$, however, ${ }^{28}$ intratympanic contrast injection provided higher perilymphatic signal compared with intravenous administration. An additional advantage of the intravenous method is simultaneous examination of both labyrinths because provided the disease manifestation is unilateral, the nondiseased ear serves for comparison. A caveat to this, however, is 
that asymptomatic EH had been observed by histopathology in $5 \%{ }^{7}$ and $26 \%{ }^{8}$ of postmortem examinations without documented MD. Finally, intravenous application of the contrast agent renders the function of the blood-perilymph barrier visible.
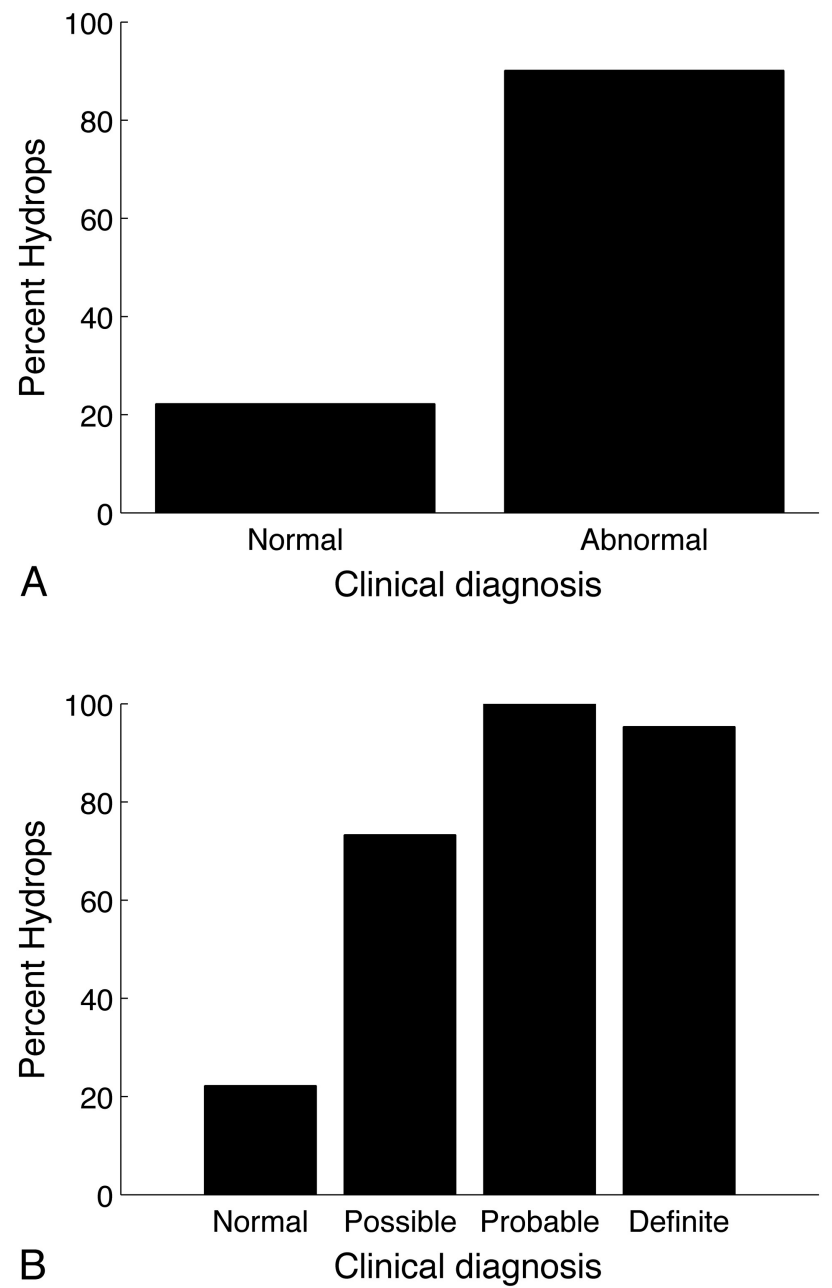

FIG 3. EH is present in $22 \%(10 / 45)$ of clinically normal ears and in $90 \%$ $(55 / 61)$ of clinically diseased ears (irrespective of clinical score) $(A)$ and in $73 \%$ of ears with possible (11/15), in $100 \%$ in ears with probable $(3 / 3)$, and in $95 \%(41 / 43)$ of ears with definite MD $(B)$.
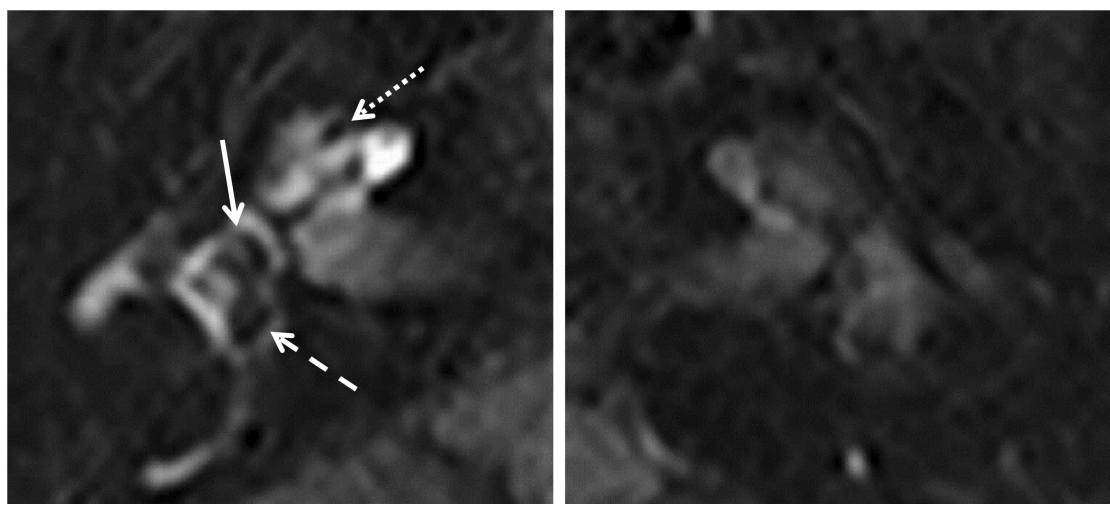

FIG 4. Predominant saccular dilation: 3D-IR sequence (right and left side of the same patient). The right side shows a dilated saccule (arrow) and a slightly distended utricle (dashed arrow) with grade I cochlear hydrops (dotted arrow). Note increased contrast enhancement of the perilymph on the symptomatic right side compared with the normal left labyrinth.
In accordance with others, ${ }^{24,28}$ gadolinium uptake was more pronounced in symptomatic compared with asymptomatic ears in patients with unilateral EH in $90 \%$ of our cases (Fig 4).

\section{Anatomic Considerations}

In 1938, Hallpike and Cairns ${ }^{29}$ described histopathologic changes that consisted of gross distention of the cochlear duct and predominant dilation of the saccule as opposed to the utricle. In most cases of our series, however, vestibular hydrops was not attributable to either the saccule or the utricle (Fig $1 B,-C$ ), and only rarely a preferential distention of the saccule (Fig 4 ) or the utricle (Fig 5) was observed. The saccule is located anteromedially in the pars inferior of the vestibule, while the utricle is posterior within the pars superior. ${ }^{30}$ Because visual assessment was based on axial images at the widest part of the vestibule (Fig 2), dilation of the vertically oriented saccule may have been underestimated in comparison with the horizontally positioned utricle. Cochlear hydrops, an apical distention of the cochlear duct as a normal finding $^{31}$ and the interscalar septum (Fig 2), can be confidently identified at this level as well.

\section{MR Imaging Grading of EH}

$\mathrm{MR}$ imaging grading of $\mathrm{EH}$ is meant to assess the presence and degree of $\mathrm{EH}$ in patients with different clinical scores of MD.

In 20 healthy volunteers, Liu et $\mathrm{al}^{32}$ found the endolymph to account for $8 \%-26 \%$ of the fluid space within the cochlea and $20 \%-41 \%$ in the vestibule. For the cochlea, Sperling et $\mathrm{al}^{33}$ proposed a grading system of $\mathrm{EH}$ with the categories "slight, moderate, and profound," based on an increasing displacement of the Reissner membrane. Visualization of the perilymphatic space within the scala vestibuli in our study is an indirect measure to depict displacement of the Reissner membrane as well. Cochlear duct dilation was relatively uniform in grade II hydrops (Fig 1C) but commonly slightly nodular in grade I (Figs $1 B$ and 4 ). Histopathologically, the existence of cochlear hydrops of varying severity in the same cochlea has been proved as well. ${ }^{34}$

The definition of a cutoff value of $>50 \%$ required for vestibular hydrops grade I was derived from $41 \%$ of the total volume encompassed by the saccule and utricle. ${ }^{33}$ Grade II was present when the entire vestibulum was occupied by the endolymph space. The MR imaging grading proposed by Nakashima et $\mathrm{al}^{34}$ probably overestimates "mild hydrops" when defined as "one-third and a half ratio" of endo- to perilymphatic space.

\section{Relationship between Clinical Diagnosis and MR Imaging Diagnosis of EH}

Belal and Antunez ${ }^{6}$ found an incidence of $\mathrm{EH}$ in $9 \%$ of 703 temporal bones, including normal ears and ears with various pathologies. By histopathology, Rauch et $\mathrm{al}^{7}$ and Merchant et $\mathrm{al}^{8}$ found $\mathrm{EH}$ in $5 \%$ and $26 \%$ of temporal bones in patients without previous symptoms of $\mathrm{MD}$. The latter figure corresponds to an 


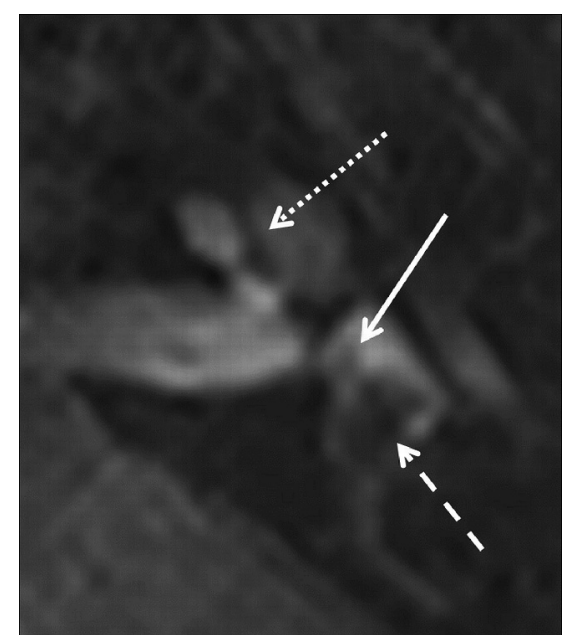

FIG 5. Predominant utricular dilation: $3 D-I R$ sequence left side. Marked distention of the utricle (dashed arrow) and sparing of the normal-sized saccule (arrow) leave the perilymphatic space visible (grade I) with no cochlear hydrops. The interscalar septum (dotted arrow) should not be mistaken for a slight cochlear hydrops.

incidence of $22 \%$ in clinically "silent" ears in our series.

$\mathrm{EH}$ was found on the clinically affected side in 55/61 ears (90\%). Histopathologically, Fraysse et al ${ }^{12}$ described similar results in $93 \%$ of 21 affected ears.

The detection rate was $73 \%$ in ears with clinically possible MD (Fig 3), 100\% in probable, and 95\% in ears with definite MD in our series. The severity of hydrops was significantly more pronounced for 55 clinically affected ears, with an average grade of $1.27 \pm 0.66$ in comparison with an average grade of $0.65 \pm 0.581$ for 10 clinically normal sides. Accordingly, Sperling et $\mathrm{al}^{33}$ found a tendency for more severe $\mathrm{EH}$ to occur in symptomatic cases and comparatively less $\mathrm{EH}$ in asymptomatic cases.

\section{CONCLUSIONS}

In patients with $\mathrm{MD}$, a dedicated $\mathrm{MR}$ imaging protocol depicts cochlear and vestibular $\mathrm{EH}$ in vivo. In accordance with histopathologic data, $10 \%$ of patients did not have $\mathrm{EH}$ in the affected ear, while EH may be present in clinically silent ears of patients with MD. A high interobserver agreement on detecting and grading $\mathrm{EH}$ suggests that this MR imaging grading method is robust.

\section{ACKNOWLEDGMENTS}

The authors thank Irini Paschalis, Laura Kopácsi, Andreas Schiller, Kathleen Sullivan, and Stefanie Schuknecht for their support of this work.

Disclosures: Bernhard Schuknecht—UNRELATED: Consultancy: Antisense Pharmaceutical Company (Regensburg, Germany), Comments: TNF $\beta$ treatment in gliomas. Stefan C.A. Hegemann-UNRELATED: Expert Testimony: SUVA, Payment for Lectures (including service on Speakers Bureaus): As a lecturer at Zurich University, I get a small amount of money for my lectures and student courses. It has absolutely nothing to do with the submitted study, Patents (planned, pending or issued): I have a patent pending (PCT/EP2013/052595), which also does not concern this study, and I still do not receive any money from it but, hopefully, will eventually, Payment for Development of Educational Presentation and Travel/Accommodations/Meeting Expenses Unrelated to Activities Listed: I sometimes give talks at conferences or courses that are supported by industrial sponsors, who also paid me some money (for my talk as well as traveling and accommodation). One sponsor I remember is Interacoustics (Drejervaenget 8, Assens, 5610. Denmark, www.interacoustics.com).

\section{REFERENCES}

1. Committee on Hearing and Equilibrium. Meniere's disease: criteria for diagnosis and evaluation of therapy for reporting. AAO-HNS Bulletin 1985;5:6-7

2. Committee on Hearing and Equilibrium guidelines for the diagnosis and evaluation of therapy in Menière's disease: American Academy of Otolaryngology-Head and Neck Foundation, Inc. Otolaryngol Head Neck Surg 1995;113:181-85

3. Greco A, Gallo A, Fusconi M, et al. Meniere's disease might be an autoimmune condition? Autoimmun Rev 2012;11:731-38

4. Lawrence M, McCabe BF. Inner-ear mechanics and deafness: special consideration of Meniere's syndrome. J Am Med Assoc 1959;171: 1927-32

5. Gates P. Hypothesis: could Meniere's disease be a channelopathy? Intern Med J 2005;35:488-89

6. Belal A Jr, Antunez JC. Pathology of endolymphatic hydrops. J Laryngol Otol 1980;94:1231-40

7. Rauch SD, Merchant SN, Thedinger BA. Meniere's syndrome and endolymphatic hydrops: double-blind temporal bone study. Ann Otol Rhinol Laryngol 1989;98:873-83

8. Merchant SN, Adams JC, Nadol JB Jr. Pathophysiology of Meniere's syndrome: are symptoms caused by endolymphatic hydrops? Otol Neurotol 2005;26:74-81

9. Berggren S. Histological investigation of three cases with Menière's syndrome. Arch Otolaryngol 1949;37:30

10. Arnvig J. Histological findings in a case of Menière's disease, with remarks on the pathologic-anatomical basis of this lesion. Acta Otolaryngol 1947;35:453-66

11. Belal A Jr, Ylikoski J. Pathologic significance of Meniere's symptom complex: a histopathologic and electron microscopic study. Am J Otolaryngol 1980;1:275-84

12. Fraysse BG, Alonso A, House WF. Menière's disease and endolymphatic hydrops: clinical-histopathological correlations. Ann Otol Rhinol Laryngol Suppl 1980;89(6 pt 3):2-22

13. Naganawa S, Ishihara S, Iwano S, et al. Three-dimensional (3D) visualization of endolymphatic hydrops after intratympanic injection of Gd-DTPA: optimization of a 3D-real inversion-recovery turbo spin-echo (TSE) sequence and application of a 32-channel head coil at 3T. J Magn Reson Imaging 2010;31:210-14

14. Schuknecht HF. Pathology of the Ear. Cambridge, Massachusetts; Harvard University Press; 1974:88

15. Niyazov DM, Andrews JC, Strelioff D, et al. Diagnosis of endolymphatic hydrops in vivo with magnetic resonance imaging. Otol Neurotol 2001;22:813-17

16. Nakashima T, Naganawa S, Sugiura M, et al. Visualization of endolymphatic hydrops in patients with Meniere's disease. Laryngoscope 2007;117:415-20

17. Naganawa S, Yamazaki M, Kawai H, et al. Visualization of endolymphatic hydrops in Menière's disease with single-dose intravenous gadolinium-based contrast media using heavily $\mathrm{T}(2)$-weighted $3 \mathrm{D}$ FLAIR. Magn Reson Med Sci 2010;9:237-42

18. Tagaya M, Teranishi M, Naganawa S, et al. 3 Tesla magnetic resonance imaging obtained 4 hours after intravenous gadolinium injection in patients with sudden deafness. Acta Otolaryngol 2010;130:665-69

19. Naganawa $S$, Yamazaki M, Kawai H, et al. Imaging of Menière's disease after intravenous administration of single-dose gadodiamide: utility of subtraction images with different inversion time. Magn Reson Med Sci 2012;11:213-19

20. Suzuki H, Teranishi M, Sone M, et al. Contrast enhancement of the inner ear after intravenous administration of a standard or double dose of gadolinium contrast agents. Acta Otolaryngol 2011;131:1025-31

21. Sano R, Teranishi M, Yamazaki M, et al. Contrast enhancement of the inner ear in magnetic resonance images taken at 10 minutes or 4 hours after intravenous gadolinium injection. Acta Otolaryngol 2012;132:241-46

22. Fukuoka H, Tsukada K, Miyagawa M, et al. Semi-quantitative eval- 
uation of endolymphatic hydrops by bilateral intratympanic gadolinium-based contrast agent (GBCA) administration with MRI for Meniere's disease. Acta Otolaryngol 2010;130:10-16

23. Naganawa S, Sugiura M, Kawamura M, et al. Imaging of endolymphatic and perilymphatic fluid at $3 \mathrm{~T}$ after intratympanic administration of gadolinium-diethylene-triamine pentaacetic acid. AJNR Am J Neuroradiol 2008;29:724-26

24. Tagaya M, Yamazaki M, Teranishi M, et al. Endolymphatic hydrops and blood-labyrinth barrier in Menière's disease. Acta Otolaryngol 2011;131:474-79

25. Carfrae MJ, Holtzman A, Eames F, et al. 3 Tesla delayed contrast magnetic resonance imaging evaluation of Menière's disease. $\mathrm{La}$ ryngoscope 2008;118:501-05

26. Fiorino F, Pizzini FB, Beltramello A, et al. Reliability of magnetic resonance imaging performed after intratympanic administration of gadolinium in the identification of endolymphatic hydrops in patients with Menière's disease. Otol Neurotol 2011;32:472-77

27. Silverstein $\mathrm{H}$, Rowan $\mathrm{PT}$, Olds MJ, et at. Inner ear perfusion and the role of round window patency. Am J Otol 1997;18:586-89

28. Yamazaki M, Naganawa S, Tagaya M, et al. Comparison of con- trast effect on the cochlear perilymph after intratympanic and intravenous gadolinium injection. AJNR Am J Neuroradiol 2012;33:773-78

29. Hallpike CS, Cairns H. Observations on the pathology of Menière's syndrome. Proc R Soc Med 1938;31:1317-36

30. Werner CF. Das Gehörorgan der Wirbeltiere und des Menschen. Beispiel für eine vergleichende Morphologie der Lagebeziehungen. Leipzig, Germany: Veb Georg Thieme; 1960:77-80

31. Yamashita T, Schuknecht HF. Apical endolymphatic hydrops. Arch Otolaryngol 1982;108:463-66

32. Liu F, Huang W, Meng X, et al. Comparison of noninvasive evaluation of endolymphatic hydrops in Meniere's disease and endolymphatic space in healthy volunteers using magnetic resonance imaging. Acta Otolaryngol 2012;132:234-40

33. Sperling NM, Paparella MM, Yoon TH, et al. Symptomatic versus asymptomatic endolymphatic hydrops: a histopathologic comparison: review. Laryngoscope 1993;103:277-85

34. Nakashima T, Naganawa S, Pyykko I, et al. Grading of endolymphatic hydrops using magnetic resonance imaging. Acta Otolaryngol Suppl 2009;560:5-8 